\title{
DERECHOS LINGÜÍSTICOS Y POLÍTICAS DE LENGUAS EN BRASIL: ESTUDIOS DE CASO EN LA UNIVERSIDAD Y LA ESCUELA PÚBLICA
}

\section{LINGUISTIC RIGHTS AND LANGUAGE POLICIES IN BRAZIL: CASE STUDIES AT THE UNIVERSITY AND PUBLIC SCHOOL}

\author{
RIMAYPAQ ALLANKAYKUNA JINASPAPAS BRASIL LLAQTAPI \\ RIMAYKUNAQ YACHANA: JATUN SUNTUR YACHAY WASIPI \\ JUCHUY YACHAYWASIPUAN ${ }^{(1)}$
}

Ligia Karina Martins de Andrade ${ }^{(2)}$ Universidad Federal de Integración Latinoamericana, Brasil

\begin{abstract}
Resumen: Este artículo analiza los derechos lingüísticos, las políticas y la planificación lingüística en dos estudios de caso, uno en la Universidad Federal de Integración Latinoamericana (UNILA) y otro en una escuela pública en la ciudad de Foz de Iguaçu. Su objetivo es observar la relación entre la legislación en Brasil y los derechos lingüísticos en contextos educativos. Utiliza una metodología de observación participante, investigación-acción, y el análisis del discurso en documentos oficiales y los producidos por indígenas e inmigrantes. La investigación propone observar y adecuar las prácticas institucionales para favorecer la diversidad, las relaciones simétricas, y mejorar el control de las políticas públicas, a favor de la equidad entre los sujetos y sus derechos lingüísticos.
\end{abstract}

Palabras clave: Derechos lingüísticos, políticas lingüísticas, subjetividad, análisis del discurso, Brasil.

Abstract: This article analyzes language rights, policies, and language planning in two case studies, one at the Federal University of Latin American Integration

(1) Traducción: Adrian T. Valer Delgado (Lengua Quechua. Cusco/Qollao)

(2) Doctora en lengua española por la USP (2009) y máster por la misma institución (2004). Profesora de lengua española adicional en la UNILA. Fue profesora de la UFAM (Manaus-Brasil). Email: ligia.andrade@unila.edu.br. 
(UNILA) and the other at a public school in Foz de Iguaçu. Its objective is to observe the relationship between legislation in Brazil and linguistic rights in educational contexts. It uses a methodology of participant observation, action research, and discourse analysis in official documents and those produced by indigenous people and immigrants. The research proposes to observe and adapt institutional practices to favor diversity, symmetrical relationships, and improve the control of public policies, in favor of equity between subjects and their linguistic rights.

Keywords: Linguistic rights, linguistic policies, subjectivity, discourse analysis, Brazil.

Ñujñu: Kay qelqasqaqa mask'an rimaypaq allankaykuna kamachikuykunata jinaspapas iskay yachanamanta, juj Jatun Suntur Yachay Wasi "Federal de Integracion Latinoamericana" (UNILA) nesqamanta jukaqtaqmi juj juchuy yachay wasi napi llaqta Foz de Iguazu. Chayanantaqmi qawanan Brasil Ilaqtaq qelqasqan kamachiykuna jinaspapas rimaypaq allankaykuna yachachina ujupi. Ruwantaqmi juj kuska qaway ñan mask'ay ruwanaq, jinaspapas camachikuy qelqakunaq mask'ay jinaspapas qeswa runakuna jamuq ruwasqampuwan. Kay mask'aytaqmi churan qawayta jinaspapas chay wasicunaq ruwanankunata lluwpaqtawan, kuskan chay tupanakunatawan, jinaspapas astawan allinchayta lluwpaq kamachikuykunata kuskanchana runakunapaq rimayninkuq allakaynimpaq.

Kichana rimaykuna: Derechos lingüísticos, políticas lingüísticas, subjetividad, análisis del discurso, Brasil. 


\section{Introducción}

Este artículo busca analizar, a partir de dos estudios de caso, la resistencia de los sujetos por medio de las lenguas y la necesidad de la defensa de los derechos lingüísticos, en el contexto de la legislación y las políticas de planificación lingüísticas adoptadas en Brasil. Se trata de un estudio de caso de una escuela pública y otro en la Universidad Federal de Integración Latinoamericana (en adelante UNILA), ambas ubicadas en la ciudad de Foz do Iguaçu (Paraná). Uno de los objetivos es subrayar que a pesar de la legislación educativa vigente y de la adhesión de Brasil a importantes tratados internacionales, todavía se necesita la implantación de medidas que garanticen los derechos lingüísticos de los grupos minorizados como forma de promover una sociedad más equitativa y plural. Sin embargo, en el ámbito de la lucha por estos derechos lingüísticos y la eficacia de políticas públicas, se señala que los sujetos imponen una resistencia y vigilancia por la concreción de estas políticas en realidades locales y regionales, pautadas por una educación intercultural, plurilingüe y diferenciada en espacios institucionales.

En primer lugar, se trata de la política pública en Brasil, en el marco de la inclusión y acceso de los indígenas, y específicamente el caso de la UNILA, a partir de un edicto de acceso (a esta Universidad) de los pueblos originarios de América Latina, lo que acompaña la Constitución de 1988 y otros documentos que promueven el acceso y derecho a la educación en todos los niveles, incluso de la enseñanza superior. En segundo lugar, se analiza la experiencia en una escuela pública en Foz de lguaçu, que se rige por legislación y exigencias regionales a partir del Mercosur (1991), dicho análisis también da pistas de este proceso sobre los sujetos y sus subjetividades en la posibilidad de cambios en las prácticas sociales y discursivas en las que están inmersos.

En el apartado 2, se presenta un recorrido histórico de la política lingüística en Brasil desde la época colonial hasta los días actuales, con el objetivo de comprender la lucha de los pueblos en el Estado brasileño, puesto que como advierte Hammel (1995, p.2) "los derechos lingüísticos forman parte de los derechos humanos fundamentales", pero no siempre se los considera de esta manera. Esta perspectiva delinea las políticas públicas actuales de inclusión de los sujetos indígenas en la educación superior en Brasil. Esto demuestra una serie de cambios a partir de la concepción de la educación como un derecho de todo(a) ciudadano(a) en el texto de la Constitución de 1988, que fue impulsada también por el movimiento social indígena y su lucha por un modelo que garantice la diversidad y su protagonismo en términos políticos, organizacionales y educacionales. Con esta iniciativa, el movimiento indígena consiguió, por primera vez en Brasil, participar de los diálogos y definir las leyes y las políticas públicas que rigen los documentos oficiales. A partir del Convenio 169 de la OIT sobre Pueblos Indígenas y Tribales (1989), de la Declaración de las Naciones Unidas sobre los Derechos de los Pueblos 
Indígenas (2007) y de la recién aprobada Declaración Americana sobre los Derechos de los Pueblos Indígenas (2016), se establece en Brasil la garantía de una educación escolar indígena de carácter bilingüe, intercultural y específica. También se relata el contexto del caso de los inmigrantes paraguayos que participaban del grupo de refuerzo escolar, lo que se verá tiene un alcance en el ámbito microsocial, no obstante, puede generar importantes efectos macrosociales.

En el apartado 2.1, se trata la cuestión del Edicto n.02/2018 que abre la selección de estudiantes indígenas en la UNILA y se considera el modelo adoptado por la universidad a partir de ese documento una innovación y conquista, lo que se observa a partir de un taller realizado de Lengua Portuguesa. Ese taller presentó algunas producciones escritas de estudiantes, lo que constituye material de análisis de este estudio, en los que se ve que la producción escrita de los estudiantes indígenas desvela la apropiación del portugués como lengua de lucha frente a la sociedad nacional. En el apartado 2.2, se discute el estudio de caso a partir de la observación participante en una escuela pública de la ciudad de Foz do lguaçu de un grupo de inmigrantes paraguayos y la necesidad de políticas que favorezcan la diversidad lingüística y cultural en las escuelas, ya que la región recibe un público inmigrante significativo. En estos dos apartados anteriores se presentan los estudios de caso y la discusión de los datos hallados, así como los resultados de la investigación y, en el último apartado, se presentan algunas conclusiones iniciales.

\subsection{Metodología}

La perspectiva metodológica interdisciplinaria adoptada en este trabajo consiste en el análisis de los estudios de caso mencionados, en los cuales los sujetos, en el primer caso indígenas y en el segundo inmigrantes paraguayos, realizan una resistencia por medio del uso de las lenguas minorizadas frente al portugués como lengua hegemónica en Brasil. En el primer caso, se analiza en el contexto de la UNILA la producción escrita de dos estudiantes indígenas de la etnia guaraní, oriundos de la región del Oeste del Paraná (Brasil), y que ingresaron a la universidad por la vía de la política de cuotas del Edicto 02/2018. Estas producciones escritas fueron divulgadas por las profesoras Laura Ferreira y Simone Cordeiro en un taller para el acompañamiento de tutores de esos estudiantes en su inserción en la universidad. La perspectiva teórica que se adopta es el análisis del discurso de las redacciones y de algunos comentarios producidos en el taller por los participantes (profesoras, estudiantes y asistencia).

En el segundo caso, el análisis se da en una perspectiva teórica de la observación participante y de la investigación acción sobre las políticas de lenguas y su planificación en la "Escola Municipal Candido Portinari" en un grupo de refuerzo escolar. El análisis de los datos recolectados se centra en la relación entre el derecho a la educación de los inmigrantes, la visibilidad de las lenguas de la 
comunidad de inmigrantes paraguayos y los documentos oficiales del Ministerio de Educación que parten de las políticas creadas en el Mercosur y que han sido observados y cotejados por la profesora coordinadora del proyecto, Ligia Andrade, en el contexto del salón de clase.

\section{La lucha por los derechos lingüísticos: la producción escrita indígena en la educación superior y la visibilidad de la(s) lengua(s) de los inmigrantes en una escuela pública de frontera}

En este apartado se contextualizan algunos aspectos relativos a la política lingüística desde la época colonial hasta los días actuales en Brasil y luego se presenta el contexto escolar de la investigación y acción realizada en el grupo de refuerzo escolar. A pesar de las diferencias, ambos casos tienen importantes similitudes en la visibilización de situaciones lingüísticas asimétricas y que pueden generar, por un lado, procesos de exclusión escolar y, por otro lado, pueden ser fuente de futuras intervenciones y planificaciones lingüísticas más acordes a las comunidades minorizadas y sus necesidades.

Desde la colonización, los jesuítas fueron los primeros que se ocuparon de la educación de los indígenas, con el fin de catequizarlos. A partir de 1549, llega a Brasil la primera misión jesuítica enviada por Don João III, compuesta por misioneros cuyo líder era el cura Manuel da Nóbrega (MEC, 2007, p.10). Debido a la resistencia de los aborígenes, los sacerdotes aprendieron las lenguas indígenas y se dedicaron a inculcar la fe cristiana, sobre todo entre los recién bautizados y los niños. Frente a dicha resistencia, eran comunes las persecuciones y la violencia empleadas contra las poblaciones nativas.

Para combatir los conflictos entre los intereses de los colonos sobre la mano de obra esclava indígena y los "no buenos ejemplos morales y religiosos para los indios" por parte de los conquistadores, los sacerdotes crearon los "aldeamentos", es decir, una especie de reducciones de indios, en los cuales, los indígenas quedaban sin contacto con el exterior, salvo si lograban autorización. En 1757, el Marqués de Pombal decreta la expulsión de los jesuitas y prohíbe el uso de la "Língua Geral", o sea, la lengua general de la mayoría de la población, pues la política lingüística de la Corona Portuguesa era la imposición del portugués como lengua hegemónica. Estas especies de reducciones (aldeamentos) se convirtieron en villas y se crean los Directorios de Indios (Diretórios de Indios). El gobernador nombraba a un director para cuidar de los indígenas, lo que no representó cambios políticos y administrativos directamente a los individuos, pero sí trajo consecuencias en la obligatoriedad de la enseñanza de la lengua portuguesa.

En 1798 se revocan los Directorios de Indios y nada los substituye hasta 1845, año en que el Decreto 426 del 24 de julio, define el Reglamento de las Misiones (Regulamento das Missões). Los sacerdotes siguen el trabajo en los "aldeamentos", 
sin embargo, ya no gozan de la autonomía anterior, y acaban sirviendo como "asistentes religiosos y educacionales" (MEC, 2007, p.12). Las escuelas se ocupaban de la instrucción primaria y se quedaban fuera de las aldeas. Además, se dedicaban al desempeño de funciones relacionadas con el "desarrollo de las provincias" y los "procesos de asimilación de la diversidad de los pueblos indígenas" (idem, p.13(3)). En todo el Período Imperial (1808-1889), hubo muchos debates para considerar el hecho de que el poder estatal se ocupara de la educación escolar primaria de negros, indios y mujeres.

El período republicano se define por la creación de una serie de escuelas técnicas que se dedican al internado de los indígenas, bajo la idea de un proyecto de asimilación e integración por medio del trabajo. De esa manera, los indígenas se convertirían en productores y consumidores de los bienes y de la tecnología producidos. En ese momento, surgen también los órganos de protección. La política lingüística de la época se tradujo en la valoración de la diversidad lingüística de los pueblos, con el propósito de utilizar las lenguas maternas en el proceso de alfabetización para grupos que no hablaban la lengua portuguesa, para facilitar el proceso de integración a la sociedad nacional (MEC, 2007, p.14), o sea, la lengua como instrumento de asimilación de los pueblos a la hegemonía nacional.

El Estado delega a institutos internacionales la educación de los indígenas, lo que implica la llegada de organizaciones religiosas de Estados Unidos a las comunidades. Se elige la enseñanza bilingüe y se producen materiales de alfabetización y capacitación para que los indígenas sean alfabetizadores en sus respectivos grupos. El modo de escolarización adoptado fue el bilingüismo de transición. En 1970, época de la dictadura militar, la Fundación Nacional del Indio (FUNAI) establece convenios con el "Summer Institute of Linguistics" (SIL), para investigar las lenguas indígenas existentes, cuyas tareas serían: identificar el sistema de sonidos, elaboración de alfabetos y análisis de las estructuras gramaticales, con el objetivo de convertir a los indígenas al protestantismo, y asumir el papel que le correspondía al Estado. Esta propuesta refuerza la política asimilacionista e integracionista del período colonial y republicano de los pueblos minorizados en la sociedad nacional.

Es a partir del debate alrededor de los derechos humanos, surgido en la década de los años 70 del siglo XX, de los procesos de descolonización, los movimientos de globalización y lucha por los derechos de las minorías, muchas veces defendiendo un tratamiento ya no como minorías, sino como grupos o naciones en el marco del Estado (Hamel, 1995), que se conquista la autodeterminación y la posibilidad de retomar la autonomía de esos pueblos. Es a partir de la "Constituição Federal" de 1988 y de la "Lei de Diretrizes e Bases da

Todas las traducciones al castellano son de la autora del artículo. 
Educação Nacional" que se fundamenta y garantiza la "Educação Escolar Indígena" en la legislación nacional. Esos documentos establecen el derecho a una educación escolar específica, diferenciada, intercultural, bilingüe/multilingüe y comunitaria, cuya competencia corresponde al Ministerio de Educación y Ciencia (MEC) de Brasil. Los pueblos indígenas participaron del debate sobre la redemocratización del país $y$, en ese entonces, lograron salir del estado de "tutela" para asumir protagonismo. El papel del Estado es ahora conocer, valorar y respetar esas formas de conocimiento tradicional. Hay varias acciones dirigidas a esos pueblos que son responsabilidad de la FUNAl, y otros órganos relacionados al gobierno, que tratan del territorio, la sostenibilidad, la salud, etc. La FUNAI se ocupa de la formulación, control y ejecución de esas acciones junto a los pueblos indígenas.

Las tres áreas de acción prioritarias de la FUNAI en el ámbito de la "Coordenação de Processos Educativos" (COPE), parte integrante de la "Coordenação Geral de Promoção da Cidadania" (CGPC) son: (1) Apoyo a la discusión y la elaboración de proyectos políticos pedagógicos; (2) Control de las políticas de educación escolar indígena; (3) Apoyo a procesos de discusión e implantación de proyectos de "Educação Profissional"; (4) Iniciativas de garantía del acceso y mantenimiento de los pueblos indígenas en la enseñanza superior.

En el ámbito de la ciudadanía, los pueblos indígenas deben tener garantizados por el Estado Democrático de Derecho, la autonomía, la autodeterminación y la consulta previa, así como poder gestionar y resolver sus conflictos a partir de soluciones internas de la comunidad, con el objetivo de garantizar la calidad de vida, la sostenibilidad, y el buen vivir de cada cultura. De acuerdo a la "Constituição Brasileira" de 1988, las directrices internacionales de derechos humanos, el Convenio OIT n. 169 sobre pueblos indígenas y tribales, los pueblos deben tener "el derecho a mantener sus propias costumbres e instituciones (...) compatibles con el sistema jurídico nacional" (Convenio 169, 1989) y deben aplicarse formas de disminución de las desigualdades históricas y sociales. Frente a esos desafíos, se escrudiña el modo como se dio la formulación de políticas de acceso a la educación superior en Brasil para suplir la desigualdad en este nivel.

En la descripción del estudio de caso de una familia de inmigrantes paraguayos en el colegio público, se introduce el esbozo del contexto de creación de los derechos y las políticas de lenguas en el ámbito regional y local. Con la creación del Mercosur, por medio del Tratado de Asunción de 1991, hubo una revisión de las políticas educacionales de los países del bloque, lo que exigió cambios en el campo de las políticas de lenguas, las cuales están directamente relacionadas con los cambios sociales y de poder. El instrumento elaborado para atender este fin se titula "Documento Marco Referencial de Desarrollo Curricular" (2001), en el cual se especifica el Programa Escuelas Interculturales Bilingües de Frontera (PEIBF) que adopta un modelo de enseñanza compartido por las escuelas participantes, 
de acuerdo con un diagnóstico sociolingüístico previo. A partir de un protocolo de intenciones de los ministros de Educación de los países miembros de "aportes en el área educacional para los objetivos políticos de integración del Mercosur" (2001, p.2), se decretó en Brasil, la Resolución n.798, del 19 de junio de 2012. Esta Resolución crea el "Programa Escuelas Interculturales Bilingües de Frontera" (PEIBF) como un programa de cooperación por parte de los Ministerios de Educación, que busca garantizar el reconocimiento y preservación de la diversidad en las regiones de frontera. Este programa tiene en cuenta los siguientes puntos: enseñanza pautada en el bilingüismo, en la interculturalidad y que garantiza a los niños y a los adolescentes la protección integral "a fin de otorgarles un desarrollo físico, mental, moral, espiritual y social en condiciones de libertad y dignidad" (Mercosur, 2001, p.2). Vale recordar que el Tratado de Asunción declara, en su artículo 23, al español, portugués y al guaraní como idiomas oficiales (idem, p.5).

Las políticas contemporáneas de creación del Mercosur impulsan una serie de medidas en los países miembros. En Brasil, se da la Ley de la Oferta de Enseñanza del Español (Ley n. 11.161, del 5 de agosto de 2005), así como en Argentina se da la obligatoriedad de la oferta en la enseñanza media del portugués, sin embargo, de carácter optativo para los estudiantes (Ley n. 26.468, del 12 de enero de 2009). Y, además, en el caso de Brasil, la Resolución n.798 trata de garantizar la articulación "de acciones que contemplen la integración regional por medio de la educación intercultural de las escuelas públicas de frontera, cambiando el ambiente escolar, y ampliando la oferta de saberes, métodos, procesos y contenidos educativos" (2001, p.2). En este sentido, los programas deben aún contemplar el fomento a la investigación, la formación y la capacitación continuada de profesionales y la elaboración de material didáctico concerniente a los intereses específicos de la región.

Este escenario político favoreció una atención a la planificación lingüística y dio margen a la antigua reivindicación en las regiones fronterizas frente a la necesidad de creación de procedimientos y procesos que contemplarán el tránsito de lenguas y sujetos. De este modo, la instauración del PEIBF cumple la función de normalizar el tránsito de profesionales en la frontera (el "cruce"), y crear una red entre las escuelas participantes del programa en diferentes países, que respete las necesidades locales. Sin embargo, pocas escuelas participan del programa y la "Escola Municipal Candido Portinari" no está contemplada. Este recorte reside en el hecho de que, a pesar de pertenecer a una región fronteriza y recibir un gran número de estudiantes inmigrantes, sobre todo paraguayos, esta escuela, y otras más, no siempre contemplan políticas adecuadas dirigidas hacia los derechos de los inmigrantes, en el sentido de la oferta de otras lenguas y culturas. Es lo que reivindica el estudio recién publicado de la UNILA sobre los derechos lingüísticos de los inmigrantes en las escuelas públicas de la ciudad (Protocolo de acolhimento de estudantes imigrantes na rede municipal de ensino) (Semed, 2020), sin embargo, 
ese protocolo defiende la enseñanza del castellano y se verá que es el guaraní paraguayo la lengua de herencia y lealtad de estos grupos.

En el próximo apartado, se discuten los casos a partir de la perspectiva teórica y metodológica interdisciplinaria adoptadas. Los resultados iniciales apuntan hacia el aporte de los individuos en espacios institucionales a la defensa de los derechos lingüísticos y la resistencia de los sujetos frente a las lenguas en la universidad y en la escuela pública.

\subsection{El Edicto $02 / 2018$ de la UNILA y las políticas nacionales de inserción en la enseñanza superior}

La UNILA fue creada por la Ley n. 12.189/2010 del presidente Luis Inacio Lula da Silva y tiene como característica central el hecho de recibir estudiantes no solo brasileños, sino también de otros países de América Latina y Caribe (UNILA, 2009). La emisión de Edictos que permiten el acceso a la universidad de los indígenas vino a sumarse a la diversidad ya vivida en la institución. Este estudio se centra en las plazas reservadas por medio del "Edicto $n^{\circ}$ 2/2018/PRAE/PROGRAD/ PROINT/REITORIA-UNILA, de 25 de julho de 2018", que contempla la selección de estudiantes indígenas de algunos países de América Latina, que puedan probar vínculo con su comunidad originaria y que vivan en sus aldeas.

En la Declaración de las Naciones Unidas sobre los Derechos de los Pueblos Indígenas (2007, p.9) consta en el artículo 21 que los pueblos tienen el derecho "al mejoramiento de sus condiciones económicas y sociales" en varias esferas (educación, salud, saneamiento, vivienda, etc.). El Estado del Paraná, en el sur de Brasil, crea el año de 2001 una ley estadual que reserva tres plazas para el ingreso de estudiantes indígenas del Estado paranaense en cada una de sus universidades estaduales. Sin embargo, en términos federales, diez años después, se promulga la Ley n.12.711, el 29 de agosto de 2012, titulada Ley de Cuotas (Lei de Cotas), por la cual el Estado brasileño adopta la política de "acciones afirmativas" dirigidas a la población negra e indígena, en universidades federales e instituciones federales de enseñanza técnica de nivel medio.

Es en este marco que varias universidades emiten edictos internos que adhieren a la creación y ejecución de la ley federal. Está claro que la ley no contempla los desafíos ni los modos de adaptación de estos sujetos a la institución. De todos modos, los números aportan datos que demuestran la importancia de la legislación, pues antes había alrededor de 50 instituciones que promovían el ingreso de estudiantes indígenas por medio de licenciaturas interculturales, cursos de formación de profesores, reserva de plazas o cupos suplementares, etc, lo que garantizó su acceso (Bergamaschi et al., 2018, p.39), en los datos de 2018, este número pasó de 1.300 plazas ocupadas en 2004, a aproximadamente 8.000 en 2012 (idem, 2018, p.40). 
La política lingüística y de planificación de la UNILA en su estatuto demuestra la laguna que los documentos dejan al mencionar muy pocas veces el término bilingüismo y bilingüe y citar mayormente los términos integración y también interdisciplinariedad (UNILA, 2009), siendo los tres términos los que forman el triplete institucional en su misión y vocación de formación de recursos humanos para el desarrollo regional y local en el ámbito del conocimiento e investigación en América Latina. Esto muestra la falta de una política lingüística clara de la institución, ya observada por los sujetos que circulaban en el espacio institucional desde el inicio, su tendencia al plurilingüismo (oficialmente, en su estatuto la universidad es bilíngüe, castellano y español) y la presencia de lenguas indígenas, debido al contingente de estudiantes paraguayos y de hablantes de lenguas indígenas de otras partes de América Latina (Diniz, 2013; Andrade, 2017), siendo el guaraní paraguayo una lengua que se caracteriza por ser de base indígena, sin embargo, es hablada por una sociedad mestiza o no indígena en Paraguay. Con el Edicto 02/2018, esta falta de política se mostró más urgente y necesaria como tema de estudio y de promoción de políticas internas.

Para analizar estos aspectos, se toma el material presentado en un taller dirigido a la comunidad académica a partir de la producción escrita en lengua portuguesa de los estudiantes indígenas. A partir de la perspectiva teórica del análisis del discurso, se observa cómo el discurso escrito en lengua portuguesa producido por los autores indígenas se convierte en lengua de lucha y resistencia frente a los procesos coloniales y a la hegemonía del portugués en el país.

Las profesoras Laura Ferreira y Simone Cordeiro en febrero de 2020, impartieron el taller de formación dirigido a la comunidad académica que acompaña a los estudiantes. En ese entonces, ellas presentaron algunas redacciones producidas por los indígenas en la disciplina de "Lengua Portuguesa Adicional" con el objetivo de reflexionar sobre la escritura académica de estos estudiantes. Vale aclarar que la universidad tiene como eje de su proyecto el bilingüismo portugués y español, lo que establece que los brasileños estudien el español y los hispanohablantes aprendan el portugués. El grupo de profesores de UNILA (Área de Lenguas) decidió que todos los indígenas ingresantes deberían tener clases de lengua portuguesa, independiente de su lengua materna, lo que en algunas etnias en Brasil coincide con la lengua hegemónica debido a los procesos de pérdida lingüística (sin embargo, estos grupos hablan y escriben lo que se asigna como la variante del portugués indígena).

En cuanto a la inserción de los indígenas en el sistema educativo, hay una preocupación por parte de los docentes del Área de Lenguas de que estos dominen la escritura académica, y que no se produzca su exclusión, sin embargo, son los individuos los que deben adaptarse al sistema universitario y su estructura, mientras que el sistema sigue con sus valores y modelos hegemónicos y excluyentes, 
como señala Baniwa (2013). La metodología expuesta en el taller y que estimuló la producción textual fue impulsada por la curiosidad de las mencionadas profesoras responsables de conocer y debatir los estereotipos en sala de clase, ya que en sus clases hay personas de varios lugares e identidades (hispanos de diversos países y también indígenas guaraníes en el caso analizado). Una de las profesoras mencionadas anteriormente sugirió el tema de los estereotipos con el objetivo de saber cuál sería la visión de los indígenas sobre los no indígenas. Este material fue analizado en el taller realizado el 2020 y debe tenerse en cuenta el hecho de que ambos autores tienen como lengua materna el guaraní, y son bilingües guaraní-portugués.

En el caso de este estudio, se analizan dos de las redacciones elaboradas, la primera de un académico y la segunda de una académica indígena. En la primera redacción, el autor indigena asocia a los no indígenas (os brancos) a la destrucción de la naturaleza, una vez que ellos se creen "los dueños de todo" (o dono de tudo) y que los indígenas son los "animales que no tienen sentimientos" (falam que os índios são os animais que não tem sentimentos) $\mathrm{y}$, al final, el argumento empleado sostiene que, en verdad, son los no indígenas "los que destruyen la naturaleza, los que son animales y los que no tienen sentimientos" (na visão dos indígenas os brancos são os destruidor da natureza). En esa producción escrita, desde el análisis del discurso que considera la relación lengua, historia e ideología, queda clara la referencia a la dicotomía utilizada en el discurso colonial en el que la colonización se justificaba debido al hecho de que los hombres que vivían en América no tenían alma, oponiéndose a los hombres civilizados, y que llegó a la evaluación peyorativa de que eran animales, bárbaros, infieles, caníbales, etc., dependiendo del interés del colonizador, pero siempre justificando la dominación sea religiosa, económica o política. Se llega a esta conclusión a partir de la oposición nosotros indígenas y ellos no indígenas y los argumentos expuestos que definen a los humanos frente a los no humanos.

En la segunda redacción, el principal argumento de la autora es el hecho de que "los blancos" tienen más dinero que los indígenas y la crítica al excesivo valor que se atribuye al dinero en la sociedad capitalista, una vez que mueran ya no serán ricos (os branco são tudo rico mesmo mas não vão levar junto quando eles morrerem só vão ficar rico até eles viverem), lo que nuevamente contrasta con la ambición y excesivo estatuto atribuido al dinero y a los objetos, a expensas de otros valores. En el caso de esa producción, hay cierta ambigüedad enunciativa que podría considerarse como cierta ironía, de doble voz, lo que crea cierta falta de hilo conductor entre los elementos del texto, en comparación al texto anterior, lo que produjo comentarios de los participantes del taller en el sentido de "no entender"el texto, considerándolo más problemático o "difícil de evaluar". Sin embargo, a pesar de algunos problemas presentados en la construcción del argumento central, el texto contiene un argumento y defiende una crítica a la sociedad de consumo y al valor del dinero como elemento principal de la vida. 
En este sentido, ambos textos presentan visiones críticas de la sociedad nacional y una resistencia a los valores considerados importantes, en el primer texto, se retoma un tema muy discutido en varias disciplinas científicas sobre la humanidad y la animalidad de los seres humanos, lo que resulta en una interesante literatura sobre las diferentes concepciones de estas nociones, por ejemplo, en la obra de Castro (1996) sobre el perspectivismo amerindio, a partir del cual no hay jerarquías entre los seres vivientes, sin embargo, la oposición retomada en la redacción vuelve a la dicotomía que justificó el dominio colonial de Europa sobre los pueblos nativos.

El análisis llevado a cabo demuestra la potencialidad de estos discursos en la esfera académica, sin profundizar el hecho de que estos sujetos puedan ocupar espacios destinados históricamente a los grupos hegemónicos, lo que parece conllevar la posibilidad de desenmascarar las formas de poder y resistir desde la escritura y en la lengua del colonizador, en este caso, el portugués. Otra observación es que los guaraníes, según los relatos de los participantes del taller, mantienen una actitud lingüística de mayor silencio en sala de clase, manifestándose cuando se les solicita, al tiempo que prefieren escribir en portugués. Se sabe que este comportamiento no corresponde en su lengua materna, el guaraní, en la cual hablan, y que también saben hablar portugués, con diferentes niveles. Esa actitud lingüística de resistencia merece un estudio aparte que consta como una observación, a pesar de su importancia. Más allá de la preocupación de que estos sujetos dominen las herramientas de instrucción académica, lo que se dará con el tiempo, se observa una importante crítica a las formas de control del poder y de las relaciones institucionales, las cuales pueden ser consideradas por la universidad en un intento de legitimar otros saberes y conocimientos para descolonizar las prácticas educacionales que mantienen la distancia en una sociedad que se perfila injusta y excluyente en Brasil.

\section{Estudio de caso del derecho lingüístico en la escuela pública "Candido Portinari"}

Este estudio parte de la observación e intervención realizada en un colegio público de Foz de Iguacu con estudiantes en refuerzo escolar. Al inicio del proyecto de extensión "Literatura e Ecocrítica na Escola", coordinado por Ligia Andrade, y financiado con recursos de la Pró-rectoría de Extensión de la UNILA, el año 2016. El aspecto observado fue cierto silencio o invisibilidad de la lengua y de la cultura españolas en la escuela, lo que debido al contingente de hispanohablantes no sería esperable. Un reciente documento realizado por un grupo de la UNILA junto a la Secretaría de Educación de la ciudad ("Protocolo de acolhimento de estudantes imigrantes na rede municipal de ensino") constató la presencia de 442 estudiantes inmigrantes inscritos en el primer ciclo (primaria). Entre ellos: 307 de Paraguay, 33 de Venezuela, 25 de Argentina, entre otras nacionalidades (Semed, 2020, p.10). A 
partir de la metodología de observación participante y de la investigación-acción, se realizaron una serie de diagnósticos e intervenciones en el contexto escolar (Elliot, 1994). A partir de ese proyecto de extensión, tal diagnóstico se dio con la observación de la profesora Ligia Andrade junto a ese grupo de estudiantes de nueve a doce años, que recibían refuerzo escolar una vez a la semana a lo largo de dos años. En el proyecto, la participación de tres hermanos paraguayos, dos niños y una niña, entre nueve y doce años de edad, se dio debido a la justificación del equipo pedagógico del colegio de que ambos menores necesitaban un refuerzo escolar. Había otros estudiantes brasileños en este grupo y la metodología adoptada en el proyecto de extensión fue la pedagogía de Freire por medio de una relación de diálogo y estímulo a la creatividad y participación de todos en el proceso de construcción del conocimiento. A partir del diagnóstico inicial, el curso de extensión propuso la introducción de varios idiomas y sus literaturas, inclusive el guaraní paraguayo; también se trabajó una serie de actividades de lecto-escritura, artes y medio ambiente, con el objetivo de influir en las relaciones del grupo por medio de la acción-investigación y de una educación liberadora dirigida a las clases populares, en la cual todos los miembros del grupo, tanto educadores como educandos, se transformaran en el proceso (Freire, 1996). La primera constatación fue que los niños en sala de clase de refuerzo y de actividades de lengua española ideadas por el curso de extensión para este contexto y grupo, no querían hablar castellano. Por parte de los alumnos brasileños, esta actitud de negación a hablar en castellano también llamó la atención. La segunda constatación cambió la manera de concebir la relación de estos inmigrantes con la lengua, como se va a explicar a continuación.

Tras las clases dictadas en guaraní por el voluntario de la UNILA, Antonio Jesús Caballero, se inició una participación más efectiva por parte de los inmigrantes, quienes se mostraron más espontáneos e interesados. La introducción del guaraní en las clases, el interés de todos (también por parte de los brasileños delante del desafío de una lengua desconocida), los esfuerzos de estudiantes, y de la profesora coordinadora que también se equivocaba, entre risas, creó una atmósfera de aprendizaje lúdica frente a las vacilaciones lingüísticas propias entre quienes están aprendiendo algo nuevo. En este contexto, quedó evidente el silenciamiento en relación a las lenguas de inmigración (castellano y guaraní) ya que el contexto escolar no favorecía el reconocimiento de la diversidad y acentuaba la falta de prácticas pedagógicas interculturales y bilingües que pudieran sumarse al rendimiento satisfactorio que se esperaba. Otro factor central es que la lengua de herencia cultural y lealtad lingüística de los inmigrantes paraguayos es el guaraní, con el que se identificaron inmediatamente en las prácticas realizadas en sala de clase.

Según Cooper (1997), la posibilidad de emplear una metodología de lo "observable" por medio de la descripción de cierto comportamiento lingüístico, y su análisis, puede generar un cambio actitudinal con relación al estatuto social de las 
lenguas en la sociedad. A partir de esa observación planteada en este estudio, se espera un cambio en el currículo acerca de la planificación lingüística y pedagógica, como la que se dio con la creación del Mercosur Educativo, por ejemplo. El mismo Cooper subraya que no se puede comprender una planificación lingüística aislada de un proceso de transformación social, pues éste está relacionado a la comunidad e impacta no solamente los problemas lingüísticos, sino también orienta un comportamiento lingüístico (p.48). Esto es central al tener en cuenta que una pequeña comunidad o grupo de hablantes son igualmente importantes para la planificación en una Nación o Estado. En el caso de los hermanos inmigrantes, se crea lo que Cooper define como una "red de comunicación", desde la cual un grupo de personas, puede ser tanto una familia como una comunidad, afectan las políticas lingüísticas del Estado, y también de los países, e incluso la forma cómo estas políticas lingüísticas pueden contemplar o silenciar grupos minoritarios. Sin embargo, el reciente estudio de la Secretaría de Educación demuestra que los inmigrantes paraguayos constituyen una diversidad que representa la mayoría en las escuelas primarias (307 paraguayos de los 442 inscritos y que respondieron a la encuesta), lo que debe ser considerado en futuras planificaciones (Semed, 2020, p.10)

Esta idea de política lingüística relacionada a la dimensión de cambio social y de poder no puede ser desconsiderada en el contexto latinoamericano contemporáneo, tal como en la creación del Mercosur y en todo su proceso de negociación como bloque económico y de integración regional macrosocial. Esto sin olvidar la reivindicación paraguaya de que el guaraní fuera considerado también como lengua oficial del bloque de todos los documentos producidos. Al relacionar la importancia de una legislación general internacional que favorezca la creación de políticas de adquisición de lenguas a la eficacia de esa implantación por el Estado, todavía se puede considerar que la planificación en esta región pasa por una creación de ciertas políticas lingüísticas. Este estudio, en otro aspecto, plantea una revisión del protagonismo de los sujetos y de la comunidad que legitiman sus reivindicaciones en el ámbito de las políticas lingüísticas microsociales. El estudio de caso desvela justamente la lucha latente de los sujetos de una comunidad, en este caso tres niños inmigrantes que toma dimensiones importantes en el cálculo de la región, lo que puede llevar a una reevaluación de las escuelas al tener en cuenta sus representaciones sociolingüísticas y su planificación frente a la comunidad escolar, lo que tiene que ver en un contexto de integración con la "inscripción de políticas lingüísticas" insertadas en una "política cultural". Según Arnoux:

Las lenguas no interpelan por su sola presencia a los individuos, es decir, no construyen subjetividades sino en la medida en que estén asociadas a discursividades que son las que les asignan sentido histórico e inciden en la conformación de las identidades colectivas. (2010, p.35).

Desde esta perspectiva, la relación entre los sujetos, sus subjetividades 
y discursividades no pueden ser ignoradas por los sectores educativos o gubernamentales, que buscan inculcar una práctica homogeneizadora, y de invisibilidad de la diversidad, que puede tener como consecuencia la exclusión escolar.

En la escuela "Candido Portinari, se observó que el cuerpo docente, frente a la situación de bilingüismo y diglosia, suele asociar el desempeño de los estudiantes a lo que sería el ideal en las competencias lingüísticas en portugués de monolingües, o sea, se les atribuye a los inmigrantes o a sus descendientes, cierta dificultad en los procesos de lectura, escritura en portugués, y también en otras disciplinas, al hecho de hablar dos o más lenguas, entre otras evaluaciones subjetivas observadas, a su vez que gran parte de los docentes no tienen una formación lingüística continua. El resultado es la participación de esos estudiantes en las clases de refuerzo escolar, una vez que se les considera con bajo o insuficiente rendimiento, lo que pasa por la adecuación de sus habilidades en la lengua portuguesa. El hecho de que gran parte de este público es hispanohablante merece atención y puede ser interpretado a la luz de la tesis de Celada (2002) que considera el español en Brasil como una lengua "singularmente estrangeira", lo que produce por parte de los brasilero(a)s cierta representación y comportamiento frente al español, de que no necesitan estudiarlo, pues se considera una "lengua próxima y fácil de entender", como afirman varios profesores y alumnos que dicen "entender todo", aunque "no la quieren hablar".

Orlandi (1997) rechaza una visión pasiva del silencio en la lengua, y lo relaciona con la historia. La autora afirma el carácter de incompletud del lenguaje evidenciado por el silencio, no como si éste ocultase un telón de fondo a ser desvelado por las palabras, sino como constituyente del decir, lo que lo hace posible:

(...) a errância dos sentidos (sua migração), a vontade do'um' (da unidade, do sentido fixo), o lugar do non sense, o equívoco, a incompletude (lugar dos muitos sentidos), do fugaz, do não apreensível), não como meros acidentes de linguagem, mas como o cerne mesmo de seu funcionamento. (1997, p.12)

En el caso analizado hay un silencio constitutivo de esta relación del sujeto entre las lenguas, y que debe moverse en el campo de las posibilidades de significación y en la fluctuación de los sentidos, y aún hay un silencio relacionado a la negación del hablar la lengua del otro (en el caso de estudiantes brasileños, el español), lo que crea una serie de barreras en las relaciones del grupo, en lo que toca, a la diversidad y cultura del otro. Esto puede dificultar la adaptación de los estudiantes paraguayos e incidir directamente en su rendimiento escolar. Al dejar de considerar este aspecto, la escuela produce una evaluación parcial y considera superficialmente como un problema lingüístico el rendimiento de estos estudiantes. 
Con lo expuesto anteriormente, se puede pensar en la experiencia de los sujetos hablantes y aproximarla a los procesos de subjetivación dentro de la nueva discursividad de la lengua que entra en el campo de batalla con la discursividad y subjetividad de la lengua materna. Eso produce un nuevo proceso de significación. El sujeto hablante se lanza en una zona de transición y asume el riesgo de cruzar a lugares de habla inéditos. Este contexto que más allá del bilingüismo, es un contexto de diglosia debido a la asimetría en los usos de las lenguas, puede favorecer la evaluación negativa por parte de los docentes que tienen ciertos criterios de desempeño establecidos desde las políticas homogeneizadoras, las cuales no consideran el papel del sujeto entre las lenguas y en la lucha con las lenguas.

\section{Conclusión}

Se puede concluir que, en ambos estudios de casos analizados, tanto en la universidad como en la escuela, hay procesos de significación que entran en juego por parte de los sujetos en la apropiación de la lengua homogeneizadora y que constituyen nuevas formas de subjetividades y discursividades en la lengua portuguesa que pasan por la criba de la lengua de las comunidades. En ambos casos, la propuesta de inserción en el sistema de educación del Estado brasileño corre el riesgo de no considerar la diversidad y producir procesos de desigualdad y exclusión. La manera como los sujetos deben adaptarse al sistema puede caracterizar una forma de exclusión más de la sociedad nacional, puesto que son los individuos los que deben adaptarse al sistema y no viceversa. Con esto no se quiere generalizar ni tampoco afirmar que la universidad o la escuela no trabajan la diversidad o las lenguas, sin embargo, esta mirada detenida sobre la cuestión lingüística y la necesidad de profundizar queda cada vez más evidenciada con la metodología expuesta en esta investigación.

Se observa que el hecho de que los documentos oficiales contemplen la diversidad no excluye la vigilancia atenta de los sujetos que, en la metodología de observación participante y análisis del discurso empleadas en este estudio, demuestra que los sujetos incorporan en sus prácticas sociales y discursivas algunas formas de resistencia tanto por medio del silencio que esconde formas de resistencia pasiva hasta la posibilidad de hablar en su lengua materna, el guaraní paraguayo en el caso de los inmigrantes. De la misma manera, la apropiación de la escritura en portugués por los académicos indígenas devela la crítica a las formas coloniales de representación del otro, y se muestran formas de resistencia por medio de la escritura en una variante del portugués indígena, las cuales merecen estudios más detallados. La incorporación del guaraní en el currículo de las escuelas públicas de la región fronteriza que se menciona, además de otras lenguas de inmigrantes como el castellano, puede constituir una práctica social y discursiva eficaz frente a las políticas lingüísticas de los grupos hegemónicos, y pueden favorecer a toda la comunidad escolar. Estas estrategias lingüísticas y pedagógicas permiten una revisión de las asimetrías en las relaciones entre los sujetos, establecen diálogos entre posibles conflictos lingüísticos silenciados, 
abren las puertas al reconocimiento de la diversidad y a relaciones más equitativas de defensa de los derechos humanos de modo pleno.

Foto 1. Texto explicativo de lo que se ve en la foto (Fuente: Ligia Andrade)

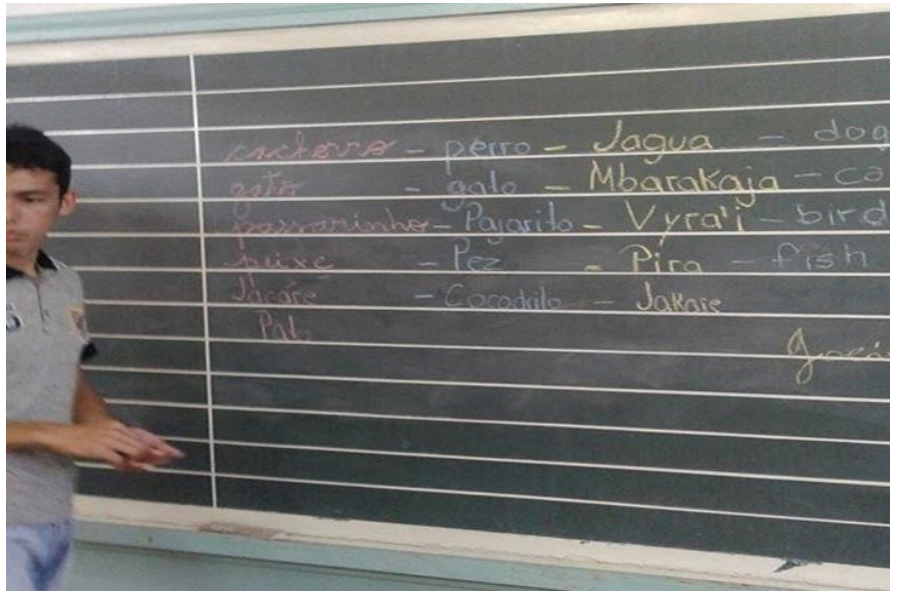

\section{Referencias}

ANDRADE, L.K.M, (2017). Material didático de espanhol como língua adicional e reflexos na leitura e escrita: um estudo de caso na UNILA. En Cácio J. Ferreira et al. Múltiplos olhares sobre o ensino de línguas: material didático, (pp.217-232). Manaus: EDUA.

ARNOUX, Elvira, (2010). Representaciones sociolingüísticas y construcción de identidades colectivas en el Mercosur. En Celada, M. et al. (Coord.). Lenguas en un espacio de integración. Acontecimientos, acciones, representaciones (pp.17-38). Buenos Aires: Biblos.

BANIWA, Gersem, (2013). A lei de cotas e os povos indígenas: mais um desafio para a diversidade. En Cadernos do Pensamento Crítico Latino-Americano (pp.1821). Brasil: CLACSO. Recuperado de: http://flacso.redelivre.org.br/files/2014/12/ XXXVcadernopensamentocritico.pdf

BERGAMASCHI, M. A. et al, (abril de 2018). Estudantes indígenas em universidades brasileira: um estudo das políticas de acesso e permanência. En Estudos RBEP (pp.37-53). Brasília, v.99, n.251. Recuperado de http://www.scielo.br/pdf/rbeped/ v99n251/2176-6681-rbeped-99-251-37.pdf

CASTRO, Eduardo Viveiros, (1996). Os pronomes cosmológicos e o perspectivismo ameríndio. En Mana (pp.115-143). Estudos de Antropologia Social. Rio de Janeiro: Apoio Fundação Universitária, José Bonifácio. Recuperado de: https://www.scielo. br/scielo.php?script=sci_arttext\&pid=S0104-93131996000200005 
CELADA, Maite, (2002). O espanhol para o brasileiro. Uma língua singularmente estrangeira. (Tesis de doctorado). Campinas: Unicamp, IEL.

CONVENÇÃO N.169 DA OIT SOBRE POVOS INDÍGENAS E TRIBAIS. (1989). (03 de febrero de 2020). Recuperado de: http://www.planalto.gov.br/ccivil_03/_ato20042006/2004/decreto/d5051.htm

COOPER, Robert L, (1997). La planificación lingüística y el cambio social. Trad. José María Perazzo. Cambridge, Inglaterra: Cambridge University Press.

DECLARACIÓN DE LAS NACIONES UNIDAS SOBRE LOS DERECHOS DE LOS PUEBLOS INDÍGENAS (2007). Naciones Unidas. Recuperado de: https://www.un.org/esa/ socdev/unpfii/documents/DRIPS_es.pdf

DINIZ, Alai, (2013). Os 'Livros Vivos' e a área de espanhol na UNILA. Intersecciones. Revista APEESP. São Paulo: Apeesp. n.1, 14-29. Recuperado de: http://www.apeesp. com.br/wp-content/uploads/14_29.pdf

ELLIOT, J, (1994). La investigación-acción en educación. España: Morata. Recuperado de http://www.terras.edu.ar/biblioteca/37/37ELLIOT-Jhon-Cap-1-y-5.pdf

FREIRE, Paulo, (1996) Pedagogia da Autonomia: saberes necessários à prática educativa. São Paulo: Paz e Terra.

HAMEL, Hainer Enrique, (1995). Derechos lingüísticos como derechos humanos: debates y perspectivas. En Alteridades. 5(10), 11-23. recuperado de: https:// alteridades.izt.uam.mx/index.php/Alte/article/view/560

MINISTÉRIO DE EDUCAÇÃO E CULTURA (MEC), (2012). Portaria n.798. Institui o Programa Escolas Interculturais de Fronteira. Brasília: MEC. Recuperado de: http:// educacaointegral.mec.gov.br

(2018). Edicto n.2/2018. Recuperado de: https:// documentos.unila.edu.br/tags/ingresso-ind-gena

(2007). Educação Escolar Indígena: diversidade sociocultural indígena ressignificando a escola. Cadernos SECAD 3. Brasília: MEC.

MERCOSUR. Escuelas de Frontera. Documento Marco Referencial de Desarrollo Curricular, (2001). Recuperado de: http://educacaointegral.mec.gov.br/escolas-defronteira

ORLANDI, E.P, (1997). As formas do silêncio: no movimento dos sentidos. Campinas: Editora da Unicamp.

SEMED, (2020). Protocolo de acolhimento a estudantes imigrantes na rede municipal de ensino. Foz do Iguacu: SEMED.

UNILA. Estatuto da UNILA, (2009). Foz do Iguaçu: UNILA, MEC. Recuperado de: https:// portal.unila.edu.br/institucional/documentos-oficiais/estatuto-da-unila/view 